\title{
Rendimiento del sector bancario en Colombia conforme a la divulgación on-line de las políticas de gobierno corporativo
}

\author{
Jairo Andrés Méndez Beltrán ${ }^{1}$ \\ Leidy Paola Acosta Contreras ${ }^{2}$ \\ Yeimi Rocío Amado Durán ${ }^{3}$
}

Recibido: 25 de julio de 2015

Aprobado: 15 de noviembre de 2015

\begin{abstract}
Méndez, J., Acosta, L. \& Amado, Y. (2015). Rendimiento del sector bancario en Colombia conforme a la divulgación on-line de las políticas de gobierno corporativo. Activos, 25, 137-166
\end{abstract}

Clasificación JEL: G21, G28.

\section{Resumen}

Dado el interés que se presenta con los temas de gobierno corporativo, este trabajo busca describir si la divulgación on-line de los contenidos de los

1 Administrador de empresas, especialista en finanzas, estudiante de la Maestría en Dirección de la Universidad del Rosario. Profesor financiero de la Universidad de La Salle, integrante del grupo de investigación Responsabilidad, rendición de cuentas y transparencia. Correo: jamendez@unisalle.edu.co

2 Economista de la Universidad de La Salle, Bogotá, Colombia. Estudiante que opta por el título de contador público de la Universidad de La Salle, Bogotá, Colombia. Correos: leidy534@yahoo.com y lacosta08@unisalle.edu.co

3 Estudiante que opta por el título de contador público de la Universidad de La Salle, Bogotá, Colombia. Correos: yeimiamado5249@hotmail.com y yamado32@ unisalle.edu.co 
códigos de buen gobierno es determinante en la generación de rendimientos sobre los activos. Para el desarrollo del trabajo se han tomado 22 entidades bancarias en Colombia en el año 2014, las cuales constituyen la población y la muestra. A partir de lo anterior, se elaboró un análisis estadístico descriptivo, con el fin de explorar si la divulgación on-line de los contenidos de los códigos de buen gobierno es determinante en la generación de rendimientos sobre los activos. Se revela que la divulgación on-line de los contenidos de los códigos de buen gobierno en las entidades bancarias en Colombia, no es determinante en el rendimiento sobre sus activos.

\section{Palabras clave}

Gobierno corporativo, divulgación de información, transparencia, retorno sobre los activos, sector bancario.

\section{Méndez, J., Acosta, L. \& Amado, Y. (2015). Performance of banking sector in Colombia according to on-line disclosure of corporate governance policies. Activos, 25, 137-166}

\section{Summary}

Given the interest that arises with corporate governance issues, this work intends to describe weather on-line disclosure of the contents of codes of good governance is crucial in generating returns on assets. For this work we analyzed 22 banks in Colombia during 2014, which were the population and the sample. Based on the above, a descriptive statistical analysis was carried out in order to explore if on-line disclosure of the contents of codes of good governance is a decisive factor in generating returns on assets. It was found that on-line disclosure of the contents of codes of good governance is not crucial in generating returns on assets.

\section{Keywords}

Corporate governance, disclosure of information, transparency, returns on assets, banking sector. 
Méndez, J., Acosta, L. \& Amado, Y. (2015). Performance du secteur bancaire en Colombie conforme à la divulgation en ligne des politiques de gouvernance des entreprises. Activos, 25, 137-166

\section{Résumé}

Compte tenu de l'intérêt qui pose les thèmes de gouvernance d'entreprise, ce document vise à décrire si la divulgation en ligne des contenus des codes de bonne gouvernance est cruciale pour générer des rendements sur les actifs. Pour le développement de ce travail 22 banques colombiennes en 2014 ont été prises en exemple, lesquels constitue la population et léchantillon. De ce qui précède, une analyse statistique descriptive a été créé, afin d'explorer si la divulgation en ligne des contenues des codes de bonne gouvernance est cruciale pour générer des rendements sur les actifs. Il est révélé que la divulgation en ligne des contenues des codes de la bonne gouvernance dans les banques colombiennes n'est pas décisive dans les rendements des actifs.

\section{Mots clés}

Gouvernance des entreprises, divulgation d'information, transparence, rendement des actifs, secteur bancaire

\section{Introducción}

Las actuaciones del cuerpo directivo de las organizaciones son cruciales en el impacto que estas podrían generar en el medio y en los resultados económicos que se esperan. El esmero de los accionistas, el consejo de administración y administradores es determinante para el desarrollo de los objetivos, en la medida en que estos actores son quienes dan los lineamientos y políticas básicas para alcanzar buenos resultados.

Colombia se ha mostrado interesada en fomentar la productividad de sus organizaciones, a través de modelos empresariales, que no solo 
brinden bienestar a los accionistas, administradores y trabajadores de las organizaciones, sino que también promuevan un entorno social más justo y equitativo. Por esta razón, la adopción del gobierno corporativo, ha sido un mecanismo para generar confianza en los usuarios, reflejándose en una mejor percepción de las organizaciones en el mercado, en aspectos de transparencia, divulgación y calidad de la información (Gaitán, 2009).

Debido a la crisis financiera que afrontó Colombia en los años noventa, se presentaron un conjunto de acontecimientos que pusieron en peligro los ahorros de millones de personas y la estabilidad económica del país (FOGAFIN, 2009). Los motivos se fundamentaban principalmente en las decisiones de las juntas directivas y de los administradores, quienes frecuentemente privilegiaban sus intereses sobre los de los accionistas, asumiendo riesgos en la búsqueda de buenos resultados.

Dado lo anterior, la falta de información transparente y confiable ha sido un factor determinante en el desarrollo de las crisis financieras por las que ha atravesado la economía del país durante la última década (Prado, 2013). Interbolsa, Fabricato, Grupo Grancolombiano, Proyectar Valores, DMG, entre otros, son ejemplos de diferentes esquemas directivos que destruyen la confianza en los grupos de interés (Vélez, 2012). La confianza resulta ser un factor definitivo al momento de alcanzar buenos resultados, en la medida en que permite generar competitividad, crear valor en las organizaciones, fomentar la oferta de empleo, adoptar mejores decisiones y reducir la percepción de riesgos en los negocios (Organización para la cooperación y el desarrollo económico, 2004).

Es por ello que la disminución de la confianza de los usuarios de las entidades bancarias, puede considerarse como un riesgo de alto impacto en la estabilidad del sistema económico del país, ya que este sector, al desempeñarse como fuente de recursos financieros, es el responsable de asignarlos a sectores de mayor rentabilidad y productividad, por lo tanto, la reducción en la inversión o en el uso de sus servicios, puede frenar el crecimiento y los desarrollos sociales y culturales del país (Estrada y Gutiérrez, 2008). 
En vista de lo anterior, mantener y mejorar la confianza de la sociedad colombiana en las entidades bancarias, a partir de la adopción de buenas prácticas de gobierno corporativo, permitiría garantizar el acceso a la información necesaria para promover la evolución económica del país (Corporación Andina de Fomento, 2010). A la vez que permitiría que las organizaciones, al ser más estables en sus procesos directivos, mejoren en cuanto a competitividad, imagen, costo de capital y liquidez (Organización para la cooperación y el desarrollo económico, 2004).

Sin embargo, los esfuerzos por implementar prácticas de buen gobierno corporativo en mercados emergentes como el colombiano, buscando generar mayor confianza y seguridad para los usuarios, presenta obstáculos como la ausencia de conocimiento sobre el gobierno corporativo en las organizaciones, los niveles de desconfianza en el mercado financiero, la falta de protección de la propiedad privada y los altos índices de riesgo en el país (Gaitán, 2009).

Por tal razón, y teniendo en cuenta su relación directa en la estabilidad del sistema financiero colombiano, se hace necesario evaluar si la información sobre gobierno corporativo publicada en la web por las entidades bancarias influye en la rentabilidad sobre los activos de estas. Lo que se espera demostrar es que debería existir una relación directa entre los niveles de transparencia, medida desde la divulgación on-line de las variables que componen los códigos de buen gobierno y los resultados en el indicador financiero Return on Assets -ROA-.

Con el fin de desarrollar esta investigación, es preciso definir el gobierno corporativo y establecer sus antecedentes en América Latina y Colombia, haciendo hincapié en la divulgación de información on-line y transparencia, la manera en que han sido adoptados por las 22 entidades bancarias vigiladas por la Superintendencia Financiera de Colombia y la influencia de estos en los niveles de confianza de sus grupos de interés.

Lo anterior se desarrolla con un análisis estadístico descriptivo, en el que se utiliza un modelo de correlación simple para cotejar si existe influencia 
en el rendimiento sobre los activos, de las 22 entidades bancarias, por la divulgación on-line de las políticas de gobierno corporativo. Asimismo se espera que este trabajo sirva como punto de partida para desarrollar futuras discusiones en el tema.

\section{Fundamentos teóricos}

\section{El gobierno corporativo y los códigos de buen gobierno del sector bancario en Colombia}

El gobierno corporativo ha evolucionado desde un concepto financiero, relacionado con el retorno de la inversión, hasta uno en el que se incluye el diseño relativo de la organización (Benavides, 2013). Esto con el fin de convertirla en un organismo de óptimo desempeño a nivel local, nacional e internacional (Superintendencia de Sociedades; Cámara de Comercio de Bogotá y Confecámaras, 2009). Con esto se podría contribuir a la estabilidad y confiabilidad en el mercado y la solidez económica del país (Bolsa de Valores de Colombia, 2009).

Uno de los debates más relevantes sobre la responsabilidad de las organizaciones, es el que inicia con el postulado de Friedman (1970). Este autor afirma que el único deber las organizaciones consiste en usar sus recursos y participar en actividades diseñadas para incrementar sus beneficios, siempre y cuando se dedique a la competencia libre y abierta, sin engaño o fraude. Posteriormente, Ross (1973) analiza el problema de agencia, que surge cuando los accionistas, denominados el principal y los administradores, conocidos como agentes, tienen diferentes objetivos y expectativas, por lo que se incrementan los costos que el principal debe asumir para monitorear las decisiones y acciones del agente.

Asimismo, Ross (1973) establece que el problema de agencia es uno de los mecanismos comúnmente codificados de interacción social. En consecuencia, la relación de agencia se apoya en tres pilares. El primero de ellos son los costos de transacción, que incluyen la obtención de información, 
negociación y cumplimiento, lo cual impide que el mercado actúe eficientemente; el segundo, la separación entre propiedad y gestión, en donde los directivos son designados por el principal para gestionar el máximo beneficio para la organización y sus accionistas; y el tercero, la asimetría de la información, la cual está relacionada con el libre mercado (Bueno y Santos, 2012).

Dado lo anterior, Jesen y Meklin (1976) postulan que en cualquier situación que involucre esfuerzos cooperativos surgen costos de agencia. Desde su publicación denominada Teoría de la firma, estos autores agrupan los costos asociados en tres partes: primero, la remuneración que le otorga el principal al agente, en virtud de un acuerdo contractual, a cambio de la defensa del interés de los accionistas y el incremento de las utilidades - costos de control-; segundo, la actuación del agente para garantizar al principal, que su gestión se orienta al cumplimiento de los objetivos de la organización -costos de fianza-; y tercero, los daños y perjuicios que debe asumir el principal, cuando el agente toma decisiones que afecten el curso normal de la organización -pérdida residual-.

Por último, Freeman (1984) y Gilbert (1984), establecen que existen grupos o individuos que pueden afectar o ser afectados por la organización en la consecución de sus objetivos. Dado lo anterior, se evidencia que el éxito de las organizaciones se debe en parte a la interdependencia con sus grupos de interés.

Esta postura permite ver a la organización desde una óptica de pluralidad de agentes, en donde no solo interactúan los propietarios y los administradores, sino que se involucran diferentes participantes, a través de contratos que dan origen a responsabilidad social empresarial (García, 2004).

Teniendo en cuenta los diversos escenarios sobre los cuales se desarrolla la economía, las organizaciones se ven en la necesidad de contar con sistemas de gobierno y de dirección eficaces. Por lo tanto, temas relacionados con la implementación del gobierno corporativo, han tomado relevancia durante los últimos años (Ganga y Vera, 2008). 
De acuerdo con la literatura existente, los conceptos más reconocidos de gobierno corporativo son: para autores como Tunzelmann (2003), entendido como la organización colectiva del proceso decisional; para Bengoechea (1996) y Canals (2004), el gobierno corporativo se relaciona con la forma en el que las organizaciones son dirigidas y controladas; mientras que para Saavedra (2008), un buen gobierno corporativo provee los mecanismos y la estructura sobre los cuales se establecen los objetivos estratégicos de la organización, los medios para alcanzarlos, así como los métodos para realizar su seguimiento y control.

Aunque actualmente existen diversos conceptos sobre gobierno corporativo, para el desarrollo de este artículo de investigación será tomado como un sistema de gestión que considera optimizar las prácticas directivas en las organizaciones, esperando mejorar la confianza de los grupos de interés y así, los resultados a futuro.

En consecuencia, la finalidad del gobierno corporativo es la de contribuir al diseño de incentivos y mecanismos de control, que permitan minimizar los costos de agencia, evitando la separación entre la propiedad y el control (Fernández y Gómez, 1999). A la vez que mejora la gestión empresarial, contribuye a la generación de valor de la organización y optimiza el acceso a los mercados financieros. Esto se alcanza con una estructura organizacional sólida, en la que se establecen códigos de ética y buenas prácticas, acciones de transparencia de la información, estructuras de sistemas de control, soluciones de controversias y el cumplimiento de la legislación vigente (Bolsa de Valores de Colombia, 2009).

Múltiples organizaciones han venido trabajando en la reducción de los conflictos de interés y en el incremento de la transparencia y la credibilidad en la información financiera. Resultado de ello, es la publicación de la Ley Sarbanes Oxley y los principios de gobernanza de la OCDE, las cuales nacen como respuesta a los mayores escándalos financieros presentados durante los años 2000 y 2002 en entidades como Xerox, Gescartera, Vivendi Universal, Dynegy, Homstore com, Global Crossing, WoldcomInc y Enron, entre otros (Jaramillo, García y Pérez, 2012). 
En el contexto latinoamericano, los primeros países en consolidar los principios de gobierno corporativo fueron México, Brasil y Argentina, seguidos por Colombia, Perú y Chile, logrando avances en flexibilización de regímenes de inversión, autorregulación en los mercados financieros, y elaboración de códigos de buen gobierno, entre otros (Organización para la cooperación y el desarrollo económico, 2004).

En la región, el proceso evolutivo del gobierno corporativo ha sido representado principalmente por el trabajo publicado en 2003, por la Mesa Redonda Latinoamericana de Gobierno Corporativo, denominado White Paper on Corporate Governance in Latin America, en la que incentiva la cooperación entre países para mejorar el cumplimiento de las normas. Al igual que el emitido en 2005 por la Corporación Andina de Fomento, hoy Banco de Desarrollo de América Latina, titulado Los lineamientos para un Código Andino de Gobierno Corporativo, con el cual contextualiza las prácticas de gobierno corporativo en empresas cerradas, medianas y pequeñas no listadas en la bolsa (Trujillo y Guzmán, 2014).

Aunque se ha permitido establecer la creación de valor en las organizaciones de cada uno de los países de América Latina, como elemento esencial para atraer inversionistas, las prácticas de gobierno corporativo se caracterizan por ser ineficientes, al limitarse a la publicación de postulados que no cumplen plenamente (Santiago y Brown, 2009). Por lo tanto, el principal problema radica en la alta concentración del control de las organizaciones por parte de los accionistas mayoritarios, desencadenando problemas de expropiación y corrupción, en los que la eficiencia y eficacia del poder judicial toma un papel trascendental en el incremento de la confianza y la credibilidad en las organizaciones (Trujillo y Guzmán, 2014).

En relación con los temas financieros, las organizaciones de América Latina tienen al sector bancario como fuente de apalancamiento para el desarrollo de sus actividades productivas (Organización para la cooperación y el desarrollo económico, 2004). Dada esta situación, aspectos como la alta concentración de propiedad accionaria, la calificación del riesgo del país, marcado por la inestabilidad política y la inoperante eficiencia de la justicia, 
han generado desconfianza en este sector (Superintendencia de Sociedades, Cámara de Comercio de Bogotá y Confecámaras, 2009).

Tabla 1. Tipos de tensiones en los que incide el problema de agencia en la economía Colombiana.

\begin{tabular}{l|l|l}
\hline Tipo de tensión & Se fundamenta en & Se refleja en \\
\hline $\begin{array}{l}\text { Entre los accionistas y los } \\
\text { administradores. }\end{array}$ & $\begin{array}{l}\text { El alto nivel de concentra- } \\
\text { ción de la propiedad en } \\
\text { pocos accionistas. }\end{array}$ & $\begin{array}{l}\text { Impide que la adminis- } \\
\text { tración asuma un control } \\
\text { considerable en la gestión } \\
\text { de la organización. }\end{array}$ \\
\hline $\begin{array}{l}\text { Entre los accionistas mayo- } \\
\text { ritarios y minoritarios. }\end{array}$ & $\begin{array}{l}\text { La alta concentración de } \\
\text { propiedad en los accionis- } \\
\text { tas mayoritarios. }\end{array}$ & $\begin{array}{l}\text { Permite que los accionistas } \\
\text { mayoritarios tomen el con- } \\
\text { trol de la organización. }\end{array}$ \\
\hline $\begin{array}{l}\text { Entre la sociedad y los } \\
\text { grupos de interés. }\end{array}$ & $\begin{array}{l}\text { La estabilidad, seguridad } \\
\text { y confianza depositada } \\
\text { por terceros en el sector } \\
\text { bancario. }\end{array}$ & $\begin{array}{l}\text { El nivel de inversión y uso } \\
\text { de los servicios ofertados } \\
\text { por los bancos. }\end{array}$ \\
\hline
\end{tabular}

Fuente: basada en información encontrada en la Superintendencia Financiera (2007).

Con el fin de dar solución a las tensiones generadas por el problema de agencia presentado en las organizaciones en Colombia, es viable revisar las ventajas, desventajas e implicaciones en Latinoamérica, a partir de la implementación de modelos de gobierno corporativo:

Tabla 2. Modelos de gobierno corporativo.

\begin{tabular}{|c|c|c|c|}
\hline Modelo & Ventajas & Desventajas & $\begin{array}{l}\text { Implicaciones en } \\
\text { Latinoamérica }\end{array}$ \\
\hline Angloamericano. & $\begin{array}{l}\text { Es muy conocido. } \\
\text { Entrega buenos } \\
\text { rendimientos. } \\
\text { Para economías } \\
\text { en auge el calor de } \\
\text { la acción aumenta } \\
\text { rápidamente. } \\
\text { Prima el accionista. }\end{array}$ & $\begin{array}{l}\text { Enfoque de corto } \\
\text { plazo: no se preocupa } \\
\text { por la permanencia } \\
\text { de la empresa al largo } \\
\text { plazo. } \\
\text { Conjunto de desco- } \\
\text { nocidos que invierten } \\
\text { junto. } \\
\text { Dirigidos por la junta } \\
\text { directiva y el gerente, } \\
\text { que buscan satis- } \\
\text { facer sus intereses } \\
\text { personales. }\end{array}$ & $\begin{array}{l}\text { Para economías } \\
\text { débiles no funciona } \\
\text { muy bien por los } \\
\text { altos costos sociales } \\
\text { que puede tener } \\
\text { asociados. } \\
\text { La cultura y los } \\
\text { valores propios de } \\
\text { los modelos son di- } \\
\text { fíciles de transmitir } \\
\text { a la cultura latina. }\end{array}$ \\
\hline
\end{tabular}




\begin{tabular}{|c|c|c|c|}
\hline Modelo & Ventajas & Desventajas & $\begin{array}{l}\text { Implicaciones en } \\
\text { Latinoamérica }\end{array}$ \\
\hline Alemán. & $\begin{array}{l}\text { Los trabajadores se } \\
\text { preocupan por la } \\
\text { corporación y, por } \\
\text { tanto, tienen menos } \\
\text { descanso laboral. } \\
\text { Visión estratégica. } \\
\text { Menos colapsos. } \\
\text { Perspectivas muy } \\
\text { atractivas para } \\
\text { el inversionista } \\
\text { extranjero. } \\
\text { Alta calidad y muy } \\
\text { competitivas. }\end{array}$ & $\begin{array}{l}\text { Los seres humanos } \\
\text { deben ser vistos } \\
\text { por igual y se } \\
\text { deben potenciar sus } \\
\text { capacidades. } \\
\text { Se pueden generar } \\
\text { conflictos entre los } \\
\text { empleados. } \\
\text { Explotación del } \\
\text { empleado. }\end{array}$ & $\begin{array}{l}\text { Manejo de los } \\
\text { sindicatos. } \\
\text { Administración y } \\
\text { participación del } \\
\text { recurso humano. }\end{array}$ \\
\hline Japonés. & $\begin{array}{l}\text { Tiene los mejores } \\
\text { elementos del } \\
\text { derecho alemán, ja- } \\
\text { ponés y americano. } \\
\text { Empleo vitalicio. } \\
\text { Prima en su orden: } \\
\text { la corporación } \\
\text { misma, los clientes, } \\
\text { empleados, acree- } \\
\text { dores y accionistas. }\end{array}$ & $\begin{array}{l}\text { Al ser colectivista no } \\
\text { hay efectividad en las } \\
\text { actividades de bajo } \\
\text { funcionamiento. }\end{array}$ & $\begin{array}{l}\text { Es atractivo para } \\
\text { los inversionistas } \\
\text { extranjeros y gene- } \\
\text { ra beneficios a los } \\
\text { grupos de interés. }\end{array}$ \\
\hline
\end{tabular}

Fuente: Gaitán (2009).

Entonces, dadas las exigencias actuales del mercado, en las que prima el desarrollo de bien común sobre el interés particular de los accionistas, la sociedad demanda en mayor medida que el comportamiento de las organizaciones integre: inteligencia y sensibilidad, ciencia y conciencia, desarrollo y sostenibilidad. Con esto se les obligaría a establecer lineamientos que liguen sus valores, creencias, ideas y voluntades, con su capacidad técnica, administrativa y financiera (Puentes, 2007). 
En vista de ello, la Superintendencia Financiera de Colombia en cooperación con ANDI, Asobancaria, Asofiduciarias, Asofondos, Bolsa de Valores de Colombia, Confecámaras y Fasecolda (2007), expiden el código de mejores prácticas corporativas, en el que estructura un conjunto de medidas sobre la asamblea de accionistas, la junta directiva, la revelación de información financiera y no financiera y la solución de controversias. Con ello, busca mitigar los riesgos administrativos, mejorar los procesos para tomar decisiones y disminuir la percepción de riesgo por parte de los usuarios, lo debería incrementar la inversión, facilitar el acceso a fuentes de financiamiento y reducir la supervisión por parte del Estado.

En dicho documento, se establece que la asamblea de accionistas, como órgano supremo del gobierno organizacional, debe determinar procedimientos para facilitar la participación de los accionistas en las asambleas y garantizar la igualdad de derechos, el trato equitativo y la comprensión de los temas a tratar. Por ello, es primordial garantizar el acceso a la información relacionada con la estructura accionaria, el orden de los temas a tratar, la conformación de la junta directiva, la aprobación de operaciones relevantes con vinculados económicos, así como los derechos y obligaciones inherentes a los accionistas.

De igual manera, a través del código de mejores prácticas corporativas (2007), se sugiere que la junta directiva, como órgano encargado de definir las principales políticas y estrategias de organización para el desarrollo de su objeto social (Deloitte Global Services Limited, 2014), esté conformado por un número impar de profesionales íntegros con perfiles que les permitan cumplir con sus funciones y cuente con un reglamento interno de funcionamiento de la junta directiva que garantice la transparencia de su gestión. Por ende, debe mantener una relación estrecha con los directores, a fin de contar con información relevante que le permita tomar decisiones adecuadas y contar con el apoyo de comités permanentes que se ocupen de temas específicos.

Ahora bien, la revelación de información financiera y no financiera, permite garantizar el derecho de inspección de los grupos de interés sobre 
la marcha y la situación de la organización, eliminando la asimetría de información. En vista de ello, el Código de mejores prácticas corporativas (2007), recomienda que la organización cuente con un medio de atención personal, telefónico o virtual que garantice la comunicación constante con los usuarios, a la vez que permita que los accionistas soliciten auditorias cuando lo consideren conveniente y puedan acceder a información sobre políticas generales, gobierno corporativo, mecanismos de control interno, contratación del revisor fiscal y cambios relevantes en el mercado, con lo cual refleja transparencia e incrementa su credibilidad.

Con respecto a la solución de controversias que se puedan presentar en las relaciones de la organización con sus accionistas y administradores, es recomendable que la organización cuente con formas alternativas de solución, tales como: la conciliación, el arbitramiento y la amigable composición. Al tiempo que brinde información sobre el procedimiento jurisdiccional para hacer efectivos sus derechos.

Infortunadamente, el sistema bancario colombiano presenta un riesgo de alto impacto en la estabilidad económica del país, al reflejar una disminución considerable en la confianza de los usuarios, que reduce la inversión, la captación de recursos y el acceso a fuentes de financiamiento. En vista de ello, la transparencia considerada como la apertura del flujo de información a datos claros, confiables y de calidad sobre el ámbito social, político y económico de la organización, permite modificar la concepción del usuario hacia una entidad accesible, competitiva y reconocida que brinda seguridad al inversor, contribuyendo en la estabilidad del mercado y velando por los derechos de los trabajadores y la comunidad en general (Corporación Andina de Fomento, 2010).

Para el desarrollo de este trabajo, se verificará la adopción de 40 aspectos de códigos de buen gobierno en las 22 entidades bancarias vigiladas por la Superintendencia Financiera de Colombia. Los aspectos a evaluar se encuentran estructurados en 5 grupos: el primero, los derechos y el trato equitativo de los accionistas compuesto por 5 variables; el segundo, la asamblea general de accionistas conformado por 10 variables; el tercero, el 
directorio compuesto por 15 variables; el cuarto, la arquitectura de control, conformado por 5 variables; y el quinto, transparencia e información financiera y no financiera compuesto por 5 variables. Los aspectos a verificar se pueden observar en la tabla 3 .

Tabla 3. Aspectos de códigos de buen gobierno.

\begin{tabular}{|c|c|}
\hline Grupo & Aspecto principal \\
\hline \multirow{5}{*}{$\begin{array}{l}\text { 1. Derechos y trato equi- } \\
\text { tativo de accionistas. }\end{array}$} & 1. Principio de paridad de trato. \\
\hline & $\begin{array}{l}\text { 2. Derecho a la no dilución de la participación en el capital } \\
\text { de la sociedad. }\end{array}$ \\
\hline & $\begin{array}{l}\text { 3. Fomento de la participación e información de los } \\
\text { accionistas. }\end{array}$ \\
\hline & 4. Cambio o toma de control por otro grupo. \\
\hline & 5. Cláusulas compromisorias de sumisión al arbitraje. \\
\hline \multirow{10}{*}{$\begin{array}{l}\text { 2. La asamblea general } \\
\text { de accionistas. }\end{array}$} & 6. Función y competencia. \\
\hline & 7. Reglamento de la asamblea general de accionistas. \\
\hline & 8. Clases y convocatoria. \\
\hline & $\begin{array}{l}\text { 9. Derecho de información de los accionistas con carácter } \\
\text { previo a la celebración de la asamblea general y durante } \\
\text { el desarrollo de la misma. }\end{array}$ \\
\hline & 10. El rol de los inversores institucionales. \\
\hline & 11. El quórum y las mayorías exigibles. \\
\hline & 12. Intervención de los accionistas. \\
\hline & 13. La regulación del derecho de voto. \\
\hline & 14. La regulación de la representación. \\
\hline & 15. Asistencia de otras personas además de los accionistas. \\
\hline
\end{tabular}




\begin{tabular}{|c|c|}
\hline Grupo & Aspecto principal \\
\hline \multirow{15}{*}{ 3. El directorio. } & 16. La necesidad de tener un directorio. \\
\hline & $\begin{array}{l}\text { 17. Atribución al directorio de las funciones de defini- } \\
\text { ción estratégica, supervisión, control, gobierno y } \\
\text { administración. }\end{array}$ \\
\hline & 18. Reglamento de directorio. \\
\hline & 19. Dimensión del directorio. \\
\hline & 20. Categorías de miembros del directorio. \\
\hline & 21. Nombramiento. \\
\hline & 22. Directores externos independientes. \\
\hline & 23. Cese de los directores. \\
\hline & $\begin{array}{l}\text { 24. Regulación de los deberes y derechos de los miembros } \\
\text { del directorio o administradores. }\end{array}$ \\
\hline & 25. Conflictos de interés y operaciones vinculadas. \\
\hline & 26. La retribución de los directores. \\
\hline & 27. La organización del directorio. \\
\hline & 28. El ejecutivo principal y alta gerencia. \\
\hline & 29. Dinámica del directorio. \\
\hline & 30. Comités del directorio. \\
\hline \multirow{5}{*}{$\begin{array}{l}\text { 4. Arquitectura de } \\
\text { control. }\end{array}$} & 31. El ambiente de control. \\
\hline & 32. La gestión de riesgos. \\
\hline & 33. El sistema de control interno. \\
\hline & $\begin{array}{l}\text { 34. La información y comunicación de la administración } \\
\text { de riesgos y sistema de control. }\end{array}$ \\
\hline & 35. Monitoreo de la arquitectura de control. \\
\hline \multirow{5}{*}{$\begin{array}{l}\text { 5. Transparencia e } \\
\text { información financiera y } \\
\text { no financiera. }\end{array}$} & 36. Política de revelación de información. \\
\hline & 37. Estados financieros. \\
\hline & 38. Información a los mercados. \\
\hline & 39. Información sobre acuerdos entre accionistas. \\
\hline & $\begin{array}{l}\text { 40. Información a incorporar en el informe anual sobre } \\
\text { gobierno corporativo. }\end{array}$ \\
\hline
\end{tabular}

Fuente: Banco de Desarrollo de América Latina (2013). 


\section{Divulgación de información on-line de las variables del gobierno corporativo}

Los principios de gobierno corporativo acerca de divulgación de datos y transparencia emitidos por la Organización para la Cooperación y el Desarrollo Económico OCDE (2004), establecen que las organizaciones deben garantizar que los canales utilizados para la divulgación de información financiera y no financiera relacionada con prácticas de gobierno corporativo, permitan un acceso igualitario, puntual y asequible para todos los usuarios (Católico, 2013). Adicionalmente, resalta la necesidad de brindar acompañamiento de personal capacitado en procesos de toma de decisiones para todos aquellos que mantengan una relación con la organización, contribuyendo así a su correcta valoración (Alonso, 2010).

En vista de ello y considerando que a diciembre de 2013 el 51,7 \% de la población colombiana tiene acceso a la web (Ministerio de Tecnologías de la Información y las Comunicaciones, 2014), las entidades bancarias han optado por el uso de la internet como uno de los canales para divulgar su información. Lo cual les permite garantizar el acceso en tiempo real a información relevante sobre hechos que afecten significativamente la posición financiera y económica de la organización, cumpliendo de esta manera con lo estipulado en los artículos 45 y 46 de la ley 222 de 1995.

Adicionalmente, divulgar información en la web promueve la transparencia real, disminuyendo la presencia de comportamientos poco éticos que afecten la estabilidad económica del país, al incrementar el costo de capital y permitir el uso inadecuado de recursos (Organización para la cooperación y el desarrollo económico, 2004). Por ello, es indispensable que la información sea elaborada conforme a políticas y prácticas contables claras, que incluyan por lo menos: estados financieros de propósito general, informes de gestión, estructura y políticas del gobierno corporativo, estructura accionaria, idoneidad de los miembros de la junta directiva y de los principales directivos, operaciones con subordinadas, posibles riesgos y planes de contingencia (Católico, 2013). 
Sin embargo, la divulgación de información vía web presenta riesgos derivados fundamentalmente de la seguridad de los sistemas. Lo cual se genera porque se pueden presentar accesos no deseados o eventuales violaciones a la información de las entidades bancarias. Esto quiere decir que, aunque existe la posibilidad de publicar un gran volumen de información en la web a bajo costo, respondiendo con las demandas del mercado, las organizaciones requieren implementar controles adecuados, que permitan a sus grupos de interés contar con información adecuada para la toma de decisiones, sin vulnerar su estabilidad (Rodríguez, 2003; Alonso, 2010).

De igual manera, debido a la cantidad de información divulgada en el mercado y la presencia de versiones contradictorias sobre las actividades de gobierno corporativo en las entidades bancarias, los usuarios cada día muestran más escepticismo sobre la información suministrada, al disminuir el nivel de inversión y el uso de los servicios ofertados por estas (Costa, 2013). Por lo tanto, para mejorar confianza de los usuarios, las organizaciones deben fortalecer sus prácticas de transparencia, los mecanismos de protección al inversionista y contribuir en la mejora del sistema judicial (Trujillo y Guzmán, 2014).

\section{Índice del retorno sobre los activos -ROA-}

El índice de retorno sobre los activos, mide la rentabilidad de una empresa con respecto a los activos que posee, por lo tanto brinda una idea de qué tan eficiente es una organización en el uso de sus activos para generar utilidades (Robles, 2012).

El cálculo del índice de retorno sobre activos, se basa en tomar la utilidad operativa y dividirla en el total de los activos de operación que posee la empresa (León, 2009). Por lo tanto, se debe tomar la utilidad operativa, es decir, aquella que la empresa obtiene de su operación básica, independiente de su estructura financiera y dividirla en los activos de operación, es decir, aquellos que se usan en el desarrollo de la actividad principal del negocio. 


$$
\text { ROA }=\frac{\text { Utilidad Operativa }}{\text { Activos de Operación) }}
$$

Expone León (2009) que el ROA es considerado uno de los indicadores apropiados para medir el éxito empresarial. Su análisis permite obtener una visión de todos los factores que influyen, ya sea de modo favorable o desfavorable, en el proceso de generación de valor de la organización. Generalmente para López (2014), este tipo de indicadores se utiliza con frecuencia para comparar resultados con otras entidades, especialmente aquellas que pertenecen al sistema bancario.

\section{Aplicación empírica}

\section{Metodología}

Para el desarrollo del trabajo se han tomado 22 entidades bancarias en Colombia en el año 2014, las cuales constituyen la población y la muestra. Con estas se realiza un estudio en el que se deduciría, si las entidades bancarias que divulgan en sus sitios web los lineamientos de sus códigos de buen gobierno, son entidades que tienen un mejor rendimiento sobre los activos.

El artículo que se presenta es de tipo exploratorio, en la medida en que los estudios encontrados tienen poca referencia hacia el problema de investigación aquí propuesto, o son muy escasos (ver tabla 4). Para este propósito se utiliza estadística descriptiva y el modelo correlacional simple, para construir una explicación que pretende servir como punto de partida para futuras investigaciones en el tema. 


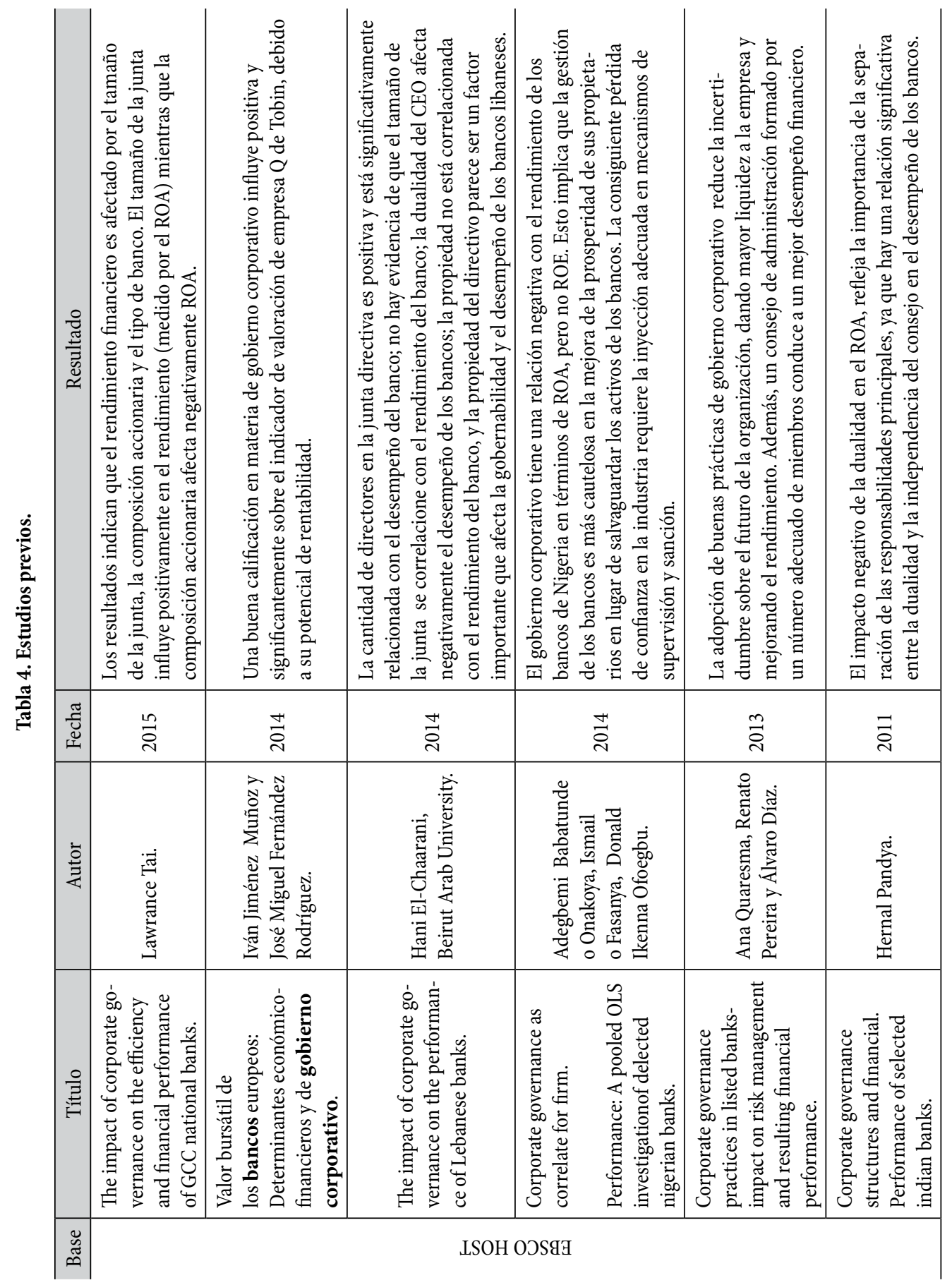

ISSN: 0124-5805, Revista Activos, N. ${ }^{\circ} 25$, julio-diciembre de 2015, pp. 137-166 
156 ACTIVOS | Jairo Andrés Méndez Beltrán, Leidy Paola Acosta Contreras, Yeimi Rocío Amado Durán

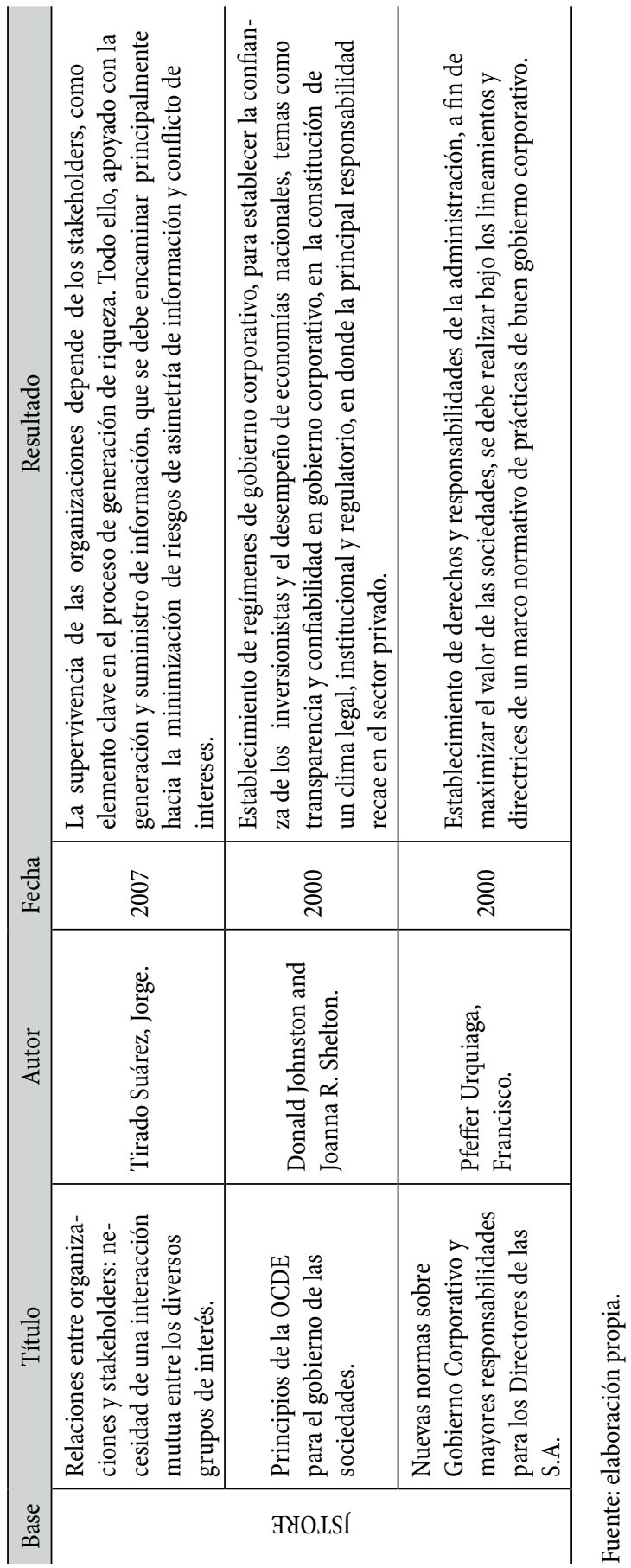

Universidad Santo Tomás, Facultad de Contaduría Pública 
El estudio consideró las 40 variables que se presentaron en la tabla 3, para evaluar la relación existente entre la divulgación de las políticas de gobierno corporativo y el rendimiento sobre los activos de las entidades bancarias en Colombia. Los cálculos de las correlaciones se realizaron con la siguiente ecuación:

$$
R=\operatorname{Correl}(x, y)=\frac{\sum(x-\bar{x})(y-\bar{y})}{\sqrt{\sum(x-\bar{x})^{2} \sum(y-\bar{y})^{2}}}
$$

En una matriz construida en una hoja de cálculo, se realizan las evaluaciones de los datos encontrados en los sitios web de cada entidad bancaria con respecto a las variables de análisis. Es así que se otorgan calificaciones de 1 para cuando el dato se encuentra, y de 0 para cuando el dato no se encuentra. Esto significa que un banco que divulga el total de las variables, obtendría una evaluación total de 40.

Al mismo tiempo, considerando que los resultados generales podrían ocultar algunos temas relevantes en el desarrollo del estudio, se realizan los cálculos de las correlaciones entre el ROA de cada entidad y cada una de las variables de la tabla 3. Entonces se construyen tablas que hacen referencia a las correlaciones positivas y negativas, para así explicar comportamientos pormenorizados de la información recolectada.

\section{Resultados y discusión}

Una vez evaluado el cumplimiento de los aspectos de los códigos de buen gobierno adoptados por las 22 entidades financieras, se presentan los resultados obtenidos en cada una de las cinco mediciones realizadas, primero, se halla la evaluación total y el porcentaje de divulgación de las políticas de gobierno corporativo; segundo, la correlación entre el ROA y la evaluación total; tercero, los descriptivos estadísticos del estudio con respecto a la evaluación total; cuarto, las variables que no tienen correlación con el ROA, y quinto, las variables con correlaciones positivas y negativas con el ROA y con algún grado de significancia. 
Tabla 5. Evaluación total y porcentaje de divulgación de las políticas de gobierno corporativo.

\begin{tabular}{r|l|c|c|c}
\hline $\mathbf{N}^{\circ}$ & Entidad bancaria & ROA & $\begin{array}{c}\text { Evaluación } \\
\text { total }\end{array}$ & $\begin{array}{c}\text { \% de cum- } \\
\text { plimiento }\end{array}$ \\
\hline 1 & Banco de Occidente S. A. & $0,01 \%$ & 35,50 & $88,75 \%$ \\
\hline 2 & Banco Falabella S. A. & $0,10 \%$ & 35,50 & $88,75 \%$ \\
\hline 3 & Banco Finandina S. A. & $0,06 \%$ & 35,50 & $88,75 \%$ \\
\hline 4 & Banco GNB Colombia S. A. & $0,57 \%$ & 35,50 & $88,75 \%$ \\
\hline 5 & Banco GNB Sudameris. & $0,08 \%$ & 35,50 & $88,75 \%$ \\
\hline 6 & Banco Pichincha S. A. & $0,09 \%$ & 35,50 & $88,75 \%$ \\
\hline 7 & Banco Popular S. A. & $0,16 \%$ & 35,50 & $88,75 \%$ \\
\hline 8 & Banco Procredit Colombia S. A. & $-0,14 \%$ & 35,50 & $88,75 \%$ \\
\hline 9 & Banco Coomeva S. A. & $0,11 \%$ & 35,50 & $88,75 \%$ \\
\hline 10 & Bancolombia S. A. & $0,15 \%$ & 34,50 & $86,25 \%$ \\
\hline 11 & Citibank Colombia S. A. & $0,29 \%$ & 34,50 & $86,25 \%$ \\
\hline 12 & Banco BBVA Colombia S. A. & $0,12 \%$ & 33,83 & $84,58 \%$ \\
\hline 13 & Banco de Bogotá. & $0,25 \%$ & 33,00 & $82,50 \%$ \\
\hline 14 & Banco Bancamía S. A. & $0,31 \%$ & 32,17 & $80,42 \%$ \\
\hline 15 & Banco Davivienda. & $0,14 \%$ & 32,00 & $80,00 \%$ \\
\hline 16 & Banco Agrario de Colombia. & $-0,03 \%$ & 27,97 & $69,92 \%$ \\
\hline 17 & Banco Caja Social. & $0,23 \%$ & 26,30 & $65,75 \%$ \\
\hline 18 & Banco WWB S. A. & $-0,10 \%$ & 26,30 & $65,75 \%$ \\
\hline 19 & Banco Cooperativo Coopcentral. & $0,04 \%$ & 26,30 & $65,75 \%$ \\
\hline 20 & Banco Comercial AV Villas S. A. & $0,15 \%$ & 22,33 & $55,83 \%$ \\
\hline 21 & Banco Corpbanca Colombia S.A. & $0,07 \%$ & 19,50 & $48,75 \%$ \\
\hline 22 & Banco Colpatria S. A. & $0,08 \%$ & 19,40 & $48,50 \%$ \\
\hline & & & & \\
\hline 12 & & \\
\hline
\end{tabular}

Fuente: elaboración propia.

La tabla 5 refleja el ROA alcanzado por cada entidad bancaria en los estados financieros reportados al 31 de diciembre de 2014, la calificación al evaluar el cumplimiento de los aspectos de códigos de buen gobierno presentados en la tabla 3 y su porcentaje total de cumplimiento. Se puede 
observar que, aunque 9 organizaciones presentan un cumplimiento del $88.75 \%$, el ROA alcanzado no es superior del 0,57 \%. Lo cual muestra la baja relación entre el cumplimiento de los aspectos evaluados en los códigos de buen gobierno y el ROA generado por cada entidad.

Tabla 6. Correlación entre el ROA y la evaluación total.

\begin{tabular}{lcc}
\hline Variables de análisis & Correlación & $\mathrm{R}^{2}$ \\
\hline ROA y evaluación total. & 0,18 & 0,03 \\
\hline
\end{tabular}

Fuente: elaboración propia.

El comportamiento del ROA se puede explicar solo en un $3 \%$ por la divulgación de políticas de gobierno corporativo. Es decir, que aunque la fundamentación teórica indica que las entidades que adoptan códigos de gobierno cumpliendo con el total de los aspectos evaluados, tienden a mejorar sus indicadores de rentabilidad, la aplicación empírica realizada en este artículo demuestra que la relación entre el ROA y el cumplimiento de los códigos de buen gobierno es muy baja.

Tabla 7. Descriptivos estadísticos del estudio con respecto a la evaluación total.

\begin{tabular}{lc}
\hline Descriptivo & Resultado \\
\hline Media. & 30,09 \\
\hline Mediana. & 34,17 \\
\hline Desviación estándar. & 5,51 \\
\hline Varianza. & 30,34 \\
\hline Mínimo. & 19,40 \\
\hline Máximo. & 35,50 \\
\hline Rango. & 16,10 \\
\hline Curtosis. & $(0,02)$ \\
\hline
\end{tabular}

Fuente: elaboración propia. 
La evaluación media del cumplimiento de los aspectos de códigos de buen gobierno, obtenido por las 22 entidades evaluadas es de 30,09; es decir, reflejan un cumplimiento promedio del $75 \%$, margen que aunque es alto, muestra la necesidad de mejorar los códigos de buen gobierno y su implementación. En tanto, la mediana, refleja que el valor central de las calificaciones obtenidas es de 34,17, pues hay 11 entidades con una calificación superior y 11 con una calificación inferior a esta. Por otra parte, la desviación estándar representa la probabilidad de que la calificación obtenida se incrementa en 5,51, aspecto que refleja la tendencia de las organizaciones por mejorar las prácticas de buen gobierno, buscando incrementar la confianza de sus grupos de interés. Asimismo, la varianza que mide la dispersión de los valores obtenidos respecto a un valor central es de 30,34.

El mínimo hace referencia a la menor calificación del cumplimiento de los aspectos de códigos de buen gobierno obtenido por las 22 entidades evaluadas, en este caso 19,40. El máximo refleja que la mayor calificación obtenida es de 35,50. Por lo tanto, el rango o diferencia entre el mínimo y máximo obtenido es de 16,10 . Esto refleja que aunque algunas entidades bancarias han realizado esfuerzos por mejorar sus códigos y prácticas de buen gobierno, otras no han adoptado códigos de buen gobierno que cumplan con los aspectos suficientes para brindar información que contribuya a mejorar la confianza de sus grupos de interés. Adicionalmente, la curtosis del 0,02 refleja un bajo grado de concentración de los valores en la región central de la distribución.

Tabla 8. Variables que no tienen correlación con el ROA.

1. Principio de paridad de trato.

2. Derecho a la no dilución de la participación en el capital de la sociedad.

3. Fomento de la participación e información de los accionistas.

10. El rol de los inversores institucionales.

29. Dinámica del directorio.

33. El sistema de control interno.

34. La información y comunicación de la administración de riesgos y sistema de control.

37. Estados financieros.

Fuente: elaboración propia. 
La tabla 8 refleja que de los 40 aspectos evaluados, 8 no tienen incidencia alguna en los resultados generados en el ROA por las 22 entidades financieras evaluadas.

La correlación entre el ROA y las variables analizadas, muestra que tan solo en el caso de las cláusulas compromisorias de sumisión al arbitraje, existe una correlación negativa, es decir, una relación inversa o decreciente.

Con las otras variables, se puede observar que aunque existe una correlación directa entre el ROA y las variables, la relación entre el indicador financiero ROA y el cumplimiento de los aspectos de códigos de buen gobierno es muy bajo.

Tabla 9. Variables con correlaciones positivas y negativas con el ROA y con algún grado de significancia.

\begin{tabular}{l|c|c}
\hline Variable & R & R2 \\
\hline 5. Cláusulas compromisorias de sumisión al arbitraje. & $(0,28)$ & 0,08 \\
\hline 7. Reglamento de la asamblea general de accionistas. & 0,27 & 0,07 \\
\hline 12. Intervención de los accionistas. & 0,17 & 0,03 \\
\hline 14. La Regulación de la representación. & 0,19 & 0,04 \\
\hline 16. La necesidad de tener un directorio. & 0,17 & 0,03 \\
\hline 18. Reglamento de directorio. & 0,20 & 0,04 \\
\hline 22. Directores externos independientes. & 0,16 & 0,03 \\
\hline 26. La retribución de los directores. & 0,18 & 0,03 \\
\hline 27. La organización del directorio. & 0,18 & 0,03 \\
\hline 28. El ejecutivo principal y alta gerencia. & 0,16 & 0,03 \\
\hline 38. Información a los mercados. & 0,18 & 0,03 \\
\hline
\end{tabular}

Fuente: elaboración propia. 
Tabla 10. Ficha técnica del estudio.

\begin{tabular}{l|l}
\hline Información & Descripción \\
\hline Población. & 22 Entidades bancarias. \\
\hline Ámbito geográfico. & Colombia. \\
\hline Fuente de información. & Sitios web de las entidades bancarias. \\
\hline Periodo analizado. & Año 2015. \\
\hline Periodo de recolección de datos. & Entre el 2 de marzo y el 9 de abril de 2015. \\
\hline Método de obtención de información. & Observación de los sitios web de cada banco. \\
\hline Tamaño de la muestra. & 22 entidades bancarias. \\
\hline Tratamiento de la información. & Análisis descriptivo. \\
\hline
\end{tabular}

Fuente. Elaboración propia.

\section{Conclusiones}

La información sobre gobierno corporativo publicada en la web por las entidades bancarias, no influye de manera significativa en la rentabilidad sobre los activos. Aunque existe una relación directa entre los niveles de transparencia, medida desde los niveles de divulgación on-line de las variables que componen los códigos de buen gobierno, los resultados en el indicador financiero ROA en cada una de las 22 entidades bancarias analizadas, demuestran que la relación entre el indicador financiero y el cumplimiento de los aspectos de códigos de buen gobierno es muy bajo.

En este sentido, se observa que aun cuando existe una relación poco significativa entre el indicador financiero ROA y el cumplimiento de los aspectos de códigos de buen gobierno para el caso de las entidades bancarias en Colombia, las prácticas de buen gobierno que se han implementado en el país durante los últimos años han permitido mejorar el control social mediante estructuras organizacionales más sólidas, el cumplimiento con legislaciones vigentes, lo cual contribuye a la gestión transparente de las entidades y al impacto que tiene cada una de sus acciones con respecto al bienestar económico de los grupos de interés. 
Por último, se destaca que aunque la información sobre gobierno corporativo publicada en la web por las entidades bancarias es necesaria para mantener informados a todos los grupos de interés, no es suficiente para establecer una relación directa con el desempeño del rendimiento sobre los activos de las entidades, y no permite modificar la concepción del usuario hacia una entidad accesible, competitiva y reconocida que brinda seguridad a sus grupos de interés.

\section{Referencias}

Alonso, M. (2010). Valoración por los analistas financieros de la información de las empresas divulgada a través de internet. Revista Estudios gerenciales, 169-190. Recuperado de http://www.icesi.edu.co/revistas/index.php/ estudios_gerenciales/article/view/336

Banco de Desarrollo de América Latina. (2013). CAF.com. Recuperado de http:// www.caf.com/es/lineamientos-gobierno-corporativo

Benavides, J. (2013). Superintendencia de Sociedades. Recuperado de http://www.supersociedades.gov.co/inspeccion-vigilancia-y-control/gobierno-corporativoy-rse/documentos/Documentos\%20RSE/Historia \%20del\%20Gobierno\%20 Corporativo\%20\%2814\%29.pdf

Bengoechea, J. (1996). El gobierno de empresas: características del debate actual. Revista de coyuntura económica, 69-118.

. (2009). Bolsa de Valores de Colombia. Recuperado de http://www. governanceconsultants.com/wp-content/uploads/2013/05/Cartilla-BVCGOBIERNO-CORPORATIVO.pdf

Bueno, J., y Santos, D. (2012). La relación de agencia, la función financiera y el gobierno corporativo. Revista de Economía y Administración, 102-108.

Canals, J. (2004). Pautas del buen gobierno en los consejos de administración. Revista Universia Business Review, 18-27.

Católico, D. (2013). Revelación en línea de la información financiera y de control en organizaciones colombianas, en el marco de la rendición de cuentas. Revista Gestión y Sociedad, 33-47.

Corporación Andina de Fomento. (2010). caf.com. Recuperado de http://www. bibliotecavirtual.info/wp-content/uploads/2013/04/GC_todo Empresario.pdf 
164 ACTIVOS | Jairo Andrés Méndez Beltrán, Leidy Paola Acosta Contreras, Yeimi Rocío Amado Durán

Costa, C. (2013). Portal Iberoamericano de marketing famaceútico. Recuperado de http://argentina.pmfarma.com/articulos/460-transparencia-credibilidad-y-confianza-la-gestion-de-la-reputacion-online-de-las-empresas-farmaceuticas.html Deloitte Global Services Limited. (2014). Deloitte. Recuperado de https://www. deloitte.com/view/es_co/co/servicios-ofrecidos/auditoria/ gobierno-corporativo/juantas-directivas/index.htm

Estrada, D., y Gutiérrez, J. (2008). Banco de la República de Colombia. Recuperado de http://www.banrep.gov.co/docum/ftp/borra490.pdf

Fernández, A., y Gómez, S. (1999). El gobierno de la empresa: mecanismos alineadores y supervisores de las actuaciones directivas. Revista Española de Financiación y Contabilidad, 355-380.

Fogafin. (2009). Crisis financiera colombiana en los años noventa. Origen, resolución y lecciones institucionales. Bogotá: Universidad Externado de Colombia.

Freeman, E. (1984). Managing for Stakeholders. Ethical Theory and Business, 57-66. Pearson.

Friedman, M. (1970). The social responsibility of business is to increase its profits. The New York Times Magazine, 2-4.

Gaitán, S. (2009). Gobierno corporativo en Colombia. Tendencias actuales. Revista Ad-minister, 137-153. Recuperado de http://publicaciones.eafit.edu.co/index. php/administer/article/download/207/258

Ganga, F., y Vera, J. (2008). El gobierno corporativo: consideraciones y cimientos teóricos. Cuadernos de adminsitración, 93-126.

García, D. (2004). Ética empresarial. Del diálogo de la confianza. España: Trotta.

Gilbert, D. (1984). La evolución del concepto Stakeholders en los escritos de Ed Freeman. IESE Business School, 1-4. Recuperado de http://www.iese.edu/es/files/La\%20evaluaci\%C3\%B3n\%20del\%20concepto\%20de\%20stakeholders\%20 seg\%C3\%BAn\%20Freeman_tcm5-39688.pdf

Jaramillo, M., García, M., y Pérez, M. (2012). Asociación Española de Contabilidad y Administración de Empresas. Recuperado de http://www.aecal.org/pub/ on_line/comunicaciones_xviicongresoaeca/cd/103d.pdf

Jensen, M., y Meckling, W. (1976). Theory of the firm: Managerial behavior, agency costs and ownership structure. University of Rochester.

León, O. (2009). Administración financiera. Fundamentos y aplicaciones. Cali: Prensa Moderna Impresores S. A. 
López, I. (2014). Expansión diccionario económico. Recuperado de http://www. expansion.com/diccionario-economico/rentabilidad-sobre-activos-roa.html Ministerio de Tecnologías de la Información y las Comunicaciones. (2014). www.mintic.gov.co. Recuperado de http://colombiatic.mintic.gov.co/602/ articles-5550_archivo_pdf

Organización para la cooperación y el desarrollo económico. (2004). www.oecd. org. Recuperado de http://www.oecd.org/daf/ca/corporategovernanceprinciples/37191543.pdf

Prado, C. (2013). asobancaria.com. Recuperado de http://www.asobancaria.com/ portal/page/portal/Eventos/eventos/XVI_CONGRESO_DE_TESORERIA/ Tab5/Cesar_Prado.pdf

Puentes, P. (2007). Transparencia en la gestión del sector empresarial. El papel del líder. Revista futuro, 17-28. Recuperado de http://www.revistafuturos.info/ raw_text/raw_futuro19/transparencia_empresarial.pdf

Robles, C. (2012). Fundamentos de administración financiera. México: Red Tercer Milenio.

Rodríguez, M. (2003). Universidad de Buenos Aires. Facultad de ciencias económicas. Recuperado de http://www.econ.uba.ar/www/institutos/contable/ centro_auditoria/trabajos/5_la_contabilidad_y_el_impacto_de_las_tecnologias_de_la_informacion_y_las_comunicaciones.pdf

Ross, S. (1973). The economic theory of agency: the principal's problem. American Economic Review, 134-139.

Santiago, M., y Brown, C. (2009). Overview of Latin American corporate governance mechanisms. Red de Revistas Cientificas de América Latina, el Caribe, España y Portugal, 26-40.

Superintendencia de Sociedades, Cámara de Comercio de Bogotá y Confecámaras. (2009). Superintendencia de Sociedades. Recuperado de http://www.supersociedades.gov.co/inspeccion-vigilancia-y-control/gobierno-corporativo-y-rse/ cartillas-y-guias/Cartillas\%20y\%20Guias/guia\%20colombiana $\% 20 \mathrm{de} \% 20$ gobierno\%20corporativo\%20\%288\%29.pdf

Superintendencia Financiera de Colombia, ANDI, Asobancaria, Asofiduciarias, Asofondos, Bolsa de Valores de Colombia. Proyecto Código País (2007). Superintendencia Financiera. Recuperado de https://www.superfinanciera. gov.co/jsp/loader.jsf?IServicio $=$ Publicaciones\&lTipo=publicaciones\&lFunci on $=$ loadContenidoPublicacion $\&$ id $=61165 \&$ dPrint $=1$ 
166 ACTIVOS | Jairo Andrés Méndez Beltrán, Leidy Paola Acosta Contreras, Yeimi Rocío Amado Durán

Trujillo, A., y Guzmán, A. (2014). Investigación sobre gobierno corporativo en Colombia. Bogotá: CESA.

Tunzelmann, N. (2003). Historical coevolution of governance and technology in the industrial revolutions. Structural Change and Economic Dynamics, 365-384.

Vélez, L. (2012). Escándalos financieros: ¿Para qué sirve el pasado? Portafolio, 8-9. Recuperado de http://www.portafolio.co/opinion/ escandalos-financieros-que-sirve-el-pasado 